\section{Retinal nerve fiber layer and macular inner retina measurements by spectral domain optical coherence tomograph in Indian eyes with early glaucoma}

HL Rao',2, JG Babu', UK Addepalli', S Senthil ${ }^{1}$ and CS Garudadri'

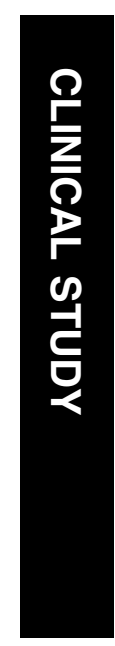

parameters were associated with large effects on the post-test probability of disease. Conclusion MIR parameters with RTVue SD-OCT were as good as the RNFL parameters to detect early glaucoma.

Eye (2012) 26, 133-139; doi:10.1038/eye.2011.277; published online 11 November 2011

Keywords: spectral domain OCT; ganglion cell complex; retinal nerve fiber layer; diagnostic accuracy

\section{Introduction}

Glaucoma is an optic neuropathy characterized by ganglion cell death that manifests clinically as characteristic optic nerve head $(\mathrm{ONH})$ and retinal nerve fiber layer (RNFL) change with correlating visual field changes. Optical coherence tomography, a well-accepted tool for glaucoma diagnosis, enables to objectively measure the ONH, RNFL and macular thickness parameters. ${ }^{1-5}$ Spectral domain OCT (SD-OCT) is a recent technique that enables the imaging of ocular structures with higher resolution and faster scan rate compared with the previous version of this technology (Stratus OCT, Carl Zeiss Meditec Inc., Dublin, CA, USA). ${ }^{6,7}$

In addition to the changes that occur in $\mathrm{ONH}$ and RNFL, another region that has been proposed to manifest changes in glaucoma is the macula, because more than $50 \%$ of the ganglion cells in the retina are located at macula and the ganglion cell layer is more than one-
${ }^{1}$ VST Glaucoma Center, LV Prasad Eye Institute, Banjara Hills, Hyderabad, India

${ }^{2}$ Center for Clinical Epidemiology and Biostatistics, LV Prasad Eye Institute, Banjara Hills, Hyderabad, India

Correspondence: HL Rao, LV Prasad Eye Institute, Banjara Hills, Hyderabad - 500034, India Tel: + 9140 30612620; Fax: + 914023548271 E-mail: harshalaxmanarao@ gmail.com or harsha@ lvpei.org

Received: 4 May 2011 Accepted in revised form: 16 September 2011 Published online: 11 November 2011

Meeting presentation: Association for Research in Vision and Ophthalmology 2010 and

American Academy of Ophthalmology 2010 
cell-layer thick at the macula. ${ }^{8,9}$ However, previous studies with Stratus OCT reported that the macular thickness measurements were inferior to RNFL measurements to detect glaucoma. ${ }^{1,2}$ One possible reason for this was that the limited resolution of Stratus OCT permitted measurements only of the entire retinal thickness, whereas the changes in glaucoma preferentially occurred in the inner retinal layers. SD-OCT by its ability to measure individual layers of the retina may capture these changes in the macula, and a few recent studies with SD-OCT, in fact, have shown that the macular inner retinal (MIR) thickness measurements, which consists of the innermost 3 layers of the retina, namely the nerve fiber layer, ganglion cell layer, and the inner plexiform layer, were as good as the RNFL measurements in detecting glaucoma. ${ }^{10-14}$ These studies, however, included glaucoma patients with all severities of visual field damage that might have biased the results, as the estimates of diagnostic accuracy are known to improve with the severity of disease. ${ }^{15-17}$ In clinical practice, the challenge is to diagnose glaucoma in early stages and there is limited literature on the diagnostic ability of SD-OCT in early glaucoma. ${ }^{17,18}$

The purpose of the present study was to compare the diagnostic accuracies of the RNFL and MIR measurements obtained with SD-OCT in early glaucoma.

\section{Methods}

This was an observational, cross-sectional, study conducted at a tertiary eye care facility in South India between August 2008 and June 2009. Informed consent was obtained from each participant, and the Ethics Committee of LV Prasad Eye Institute approved all methodology. All methods adhered to the tenets of the Declaration of Helsinki for research involving human subjects.

Consecutive glaucoma patients, categorized as early stage by Hodapp-Anderson-Parrish classification ${ }^{19}$ formed the study group, whereas the normal subjects were recruited from among those who came for a routine eye examination, patients' relatives and hospital staff. Inclusion criteria were age $\geq 18$ years, best corrected visual acuity of 20/40 or better, refractive error within $\pm 5.0 \mathrm{D}$ sphere and $\pm 3 \mathrm{D}$ cylinder, and willingness to participate in the study. Exclusion criteria were presence of any media opacities that prevented good imaging, intraocular surgery within the previous 6 months, any retinal (including macular) or neurological diseases other than glaucoma that could confound the results of visual field examination and structural measurements with SD-OCT.
All participants underwent a comprehensive ocular examination that included a detailed medical history, best corrected visual acuity measurement, slit-lamp biomicroscopy, Goldmann applanation tonometry, gonioscopy, dilated fundus examination, standard automated perimetry (SAP), and SD-OCT imaging with RTVue (Optovue Inc, Fremont, CA, USA).

Control subjects had a normal ocular examination, intraocular pressure (IOP) less than $22 \mathrm{~mm} \mathrm{Hg}$ in both eyes, no past history of increased IOP, no family history of glaucoma, no optic disc morphology suspicious of glaucoma, and normal visual fields. Optic disc examinations were done by two glaucoma specialists, and glaucomatous damage was suspected on the basis of focal or diffuse neuroretinal rim thinning, localized notching or nerve fiber layer defects. Glaucoma patients had characteristic optic disc changes, as defined above, with correlating visual field changes. All glaucoma patients had previous experience of undergoing visual field examination.

SAP was performed using a Humphrey Field analyzer, model 750 (Zeiss Humphrey Systems, Dublin, CA, USA), with the Swedish interactive threshold algorithm standard 24-2 algorithm. Reliability criteria were xation losses and false positive or false negative response rates of less than $20 \%$. Abnormal SAP results were considered to be related to glaucoma, if they correlated with the optic disc findings and there were no other abnormalities to explain the defect. Glaucomatous visual eld defects were dened by two of the following three criteria on SAP: the presence of a cluster of three points on pattern deviation probability plot with a $P$-value of less than $5 \%$, one of which had a $P$-value less than $1 \%$, or a pattern standard deviation with a $P$-value less than $5 \%$, or a glaucoma hemield test result outside normal limits.

\section{Instrumentation}

SD-OCT examination was performed with the RTVue (software version 4.0.5.39; Optovue Inc., Fremont, CA, USA). RTVue uses a scanning laser diode with a wavelength of $840 \pm 10 \mathrm{~nm}$ to provide images of ocular microstructures. The protocols used for imaging with RTVue in this study were ONH and GCC (ganglion cell complex). These protocols have been explained earlier. ${ }^{11}$ All patients had both protocols performed on the same day.

\section{Optic nerve head scan}

The $\mathrm{ONH}$ protocol consists of 12 radial scans, $3.4 \mathrm{~mm}$ in length and 6 concentric ring scans ranging from 2.5 to $4.0 \mathrm{~mm}$ in diameter all centered on the optic disc. Retinal pigment epithelium (RPE) tips were automatically 
detected by the software, which were refined manually by the operator. The RTVue software, then, delineates the optic disc margin by joining the RPE tips. The optic cup is automatically defined by the software by fitting a plane $150 \mu \mathrm{m}$ parallel to and above a plane that fits the coordinates of the RPE tips by the least squared error method. ONH protocol calculates various parameters that describe the $\mathrm{ONH}$ and also generates a polar RNFL thickness map that is the RNFL thickness measured along a circle $3.45 \mathrm{~mm}$ in diameter centered on the optic disc. The parameters generated are the average RNFL thickness in the temporal, superior, nasal, and inferior quadrant as well as the overall average along the entire measurement circle. In addition, the superior, inferior, and the overall average measurements are compared with the normative database within the software and a diagnostic categorization is provided as outside normal limits, if the value falls lower than the $99.9 \%$ confidence interval (CI) of the healthy, age-matched population. A borderline result indicates that the value is between the $95 \%$ and $99.9 \% \mathrm{CI}$, and a within-normal-limits result indicates that the value is within the $95 \% \mathrm{CI}$. The normative database of RTVue has data on 1081 subjects of different ethnicities, which are used to derive the normal, borderline, and outside normal limits cutoffs for these parameters.

\section{GCC scan}

The GCC protocol was used to obtain MIR measurements. The parameters generated by the GCC analysis are the average, superior, and inferior MIR thickness, average superior-minus inferior-MIR thickness and superior-minus-inferior thickness standard deviation. In addition to the above parameters, the GCC protocol provides three other parameters called global loss volume (GLV), focal loss volume (FLV) and root mean square (RMS). These have been explained previously. ${ }^{10,11}$ GLV measures the average amount of GCC loss over the entire GCC map. It is calculated from the fractional deviation map, which is the map showing the percentage of GCC thickness decreases at each pixel location compared with the expected or normal value at each pixel determined by the instrument's built-in normative database. GLV is calculated by dividing the sum of the values at all pixel locations where the fractional deviation map value is $<0$, by the total area. FLV measures the average amount of focal loss over the entire GCC map. FLV detects focal loss using a pattern deviation map to correct for the overall absolute changes, much like the corrected pattern standard deviation in the visual fields. For calculation of FLV, a pattern map, which is a normalized map calculated by dividing the GCC thickness values at each location by the average GCC thickness value from the entire map (for an individual), is calculated. The difference between this pattern map for an individual and the average pattern map of the normative database gives the pattern deviation map. A probability value is assigned at each pixel depending on the statistical significance of this difference. FLV is calculated by dividing the sum of the pattern deviation map values at pixels where the fractional deviation map value is $<0$ and the pattern deviation map value is statistically significant at $P<5 \%$, by the total area. RMS or the pattern coefficient of variation provides a summary of how well the fractional and pattern deviation maps of an individual fit the normal pattern. The worse the fit, the higher the value. In addition, the average, superior, and inferior MIR thickness, FLV and GLV are compared with the normative database within the software and a diagnostic categorization is provided as outside normal limits, borderline, and within normal limits similar to the RNFL measurements. All GCC scans were inspected for segmentation algorithm failures and one eye in glaucoma group where the algorithm failed was excluded.

Only good quality images, as defined by a signal strength index of $\geq 30$ were used for analysis. RTVue imaging was done on the same day as visual field testing.

\section{Statistical analysis}

Descriptive statistics included mean and standard deviation for normally distributed variables, and median, first quartile, and third quartile values for nonnormally distributed variables. Receiver operating characteristic (ROC) curves were used to describe the ability to discriminate glaucomatous from healthy eyes for each RTVue software-provided parameter.

Sensitivities at fixed specificities of $80 \%$ and $95 \%$ were determined for all the parameters. To obtain CIs for areas under the receiver operating characteristic curves (AUC), a bootstrap re-sampling procedure was used $(n=1000$ re-samples). As measurements from both eyes of the same subject are likely to be correlated, the cluster of data for the study subject was considered as the unit of re-sampling when calculating standard errors. This procedure has been used in literature to adjust for the presence of multiple correlated measurements from the same unit. ${ }^{20,21}$

Likelihood ratios (LRs) were reported for diagnostic categorization (outside normal limits, borderline, or within normal limits) provided by the instrument after comparison with the respective normative database. LR is the probability of a given test result in those with disease divided by the probability of the same test result in those without the disease. ${ }^{22-24}$ The LR for a given test result indicates how much that result will raise or lower 
the probability of disease. A LR of 1 or close to 1 would mean that the test provides no further information about the post-test probability of the disease. LRs higher than 10 or lower than 0.1 would be associated with large effects on post-test probability; LRs from 5 to 10 or from 0.1 to 0.2 would be associated with moderate effects; LRs from 2 to 5 or from 0.2 to 0.5 would be associated with small effects. ${ }^{22}$ The $95 \%$ CIs for LRs were calculated according to the method proposed by Simel et al. ${ }^{25}$

Statistical analyses were performed using commercial software (Stata ver. 10.0; StataCorp, College Station, TX, USA). A $P$-value of $<0.05$ was considered statically significant.

\section{Results}

In all, 125 eyes of 64 normal subjects and 91 eyes of 59 early glaucoma patients were included for the analysis. Age and SAP parameters of the two groups of participants are shown in Table 1. Patients in the glaucoma group had worse mean deviation and pattern standard deviation on SAP compared with the normal subjects.

The mean values of the RNFL parameters in the two groups of participants are shown in Table 2. Significant differences between the two groups were found for all the parameters except the temporal quadrant RNFL thickness. AUC and sensitivities at fixed specificities for the parameters are also shown in Table 2. The AUCs for the RNFL parameters ranged from 0.537 for the temporal quadrant thickness to 0.821 for the

Table 1 Age and visual field parameters of the study participants

\begin{tabular}{lccc}
\hline & $\begin{array}{c}\text { Normal subject } \\
\text { group (125 eyes } \\
\text { of } 64 \text { subjects) }\end{array}$ & $\begin{array}{c}\text { Early glaucoma } \\
\text { group (91 eyes } \\
\text { of 59 patients) }\end{array}$ & P-value \\
\hline Age (years) & $47.7 \pm 13.4$ & $51.8 \pm 13.4$ & 0.09 \\
MD (dB) & $-1.3 \pm 1.2$ & $-2.6 \pm 1.8$ & $<0.001$ \\
PSD (dB) & $1.6 \pm 0.3$ & $2.4 \pm 1.5$ & $<0.001$ \\
\hline
\end{tabular}

Abbreviations: $\mathrm{MD}$, mean deviation; $\mathrm{PSD}$, pattern standard deviation; $\mathrm{dB}$, decibel (all values reported as mean $\pm \mathrm{SD}$ ) inferior quadrant RNFL thickness. Inferior quadrant thickness had the highest sensitivity of $58.2 \%$ at a specificity of $95 \%$.

The mean values of the MIR measurements in the two groups of participants are shown in Table 3. Significant differences between the two groups were found for all the parameters. AUC and sensitivities at fixed specificities for the parameters are also shown in Table 3. The AUCs for the MIR parameters ranged from 0.603 for the superior-minus-inferior thickness averaged to 0.908 for FLV. FLV also had the largest sensitivity of $52.7 \%$ at a specificity of $95 \%$.

Table 4 shows the LRs with their 95\% CIs for the parameters that are compared with the instrument's normative database. For most of the parameters except FLV and GLV, outside normal limits results were associated with large effects on the post-test probability of disease. Borderline results were associated with small effects, whereas, within normal limits, results were associated with no effects on the post-test probability of disease.

Figure 1 shows the ROC curves of the best RNFL and the best MIR parameter. The AUC of the MIR parameter with the largest AUC, FLV, was significantly higher than that of the RNFL parameter with the largest AUC, inferior quadrant RNFL thickness (0.908 vs 0.821 ; $P<0.001)$. The sensitivities of these parameters at high specificity of $95 \%$, however, appeared comparable (52.7 vs. $58.2 \%$ ). Figure 2 shows the ROC curves of the average MIR and average RNFL thickness parameters that were also comparable (0.735 vs. $0.799 ; P=0.07)$.

\section{Discussion}

In this study, we demonstrated that the MIR parameters were as good as the RNFL parameters to detect early glaucoma in Indian eyes. We also reported the LRs of the diagnostic classification of the parameters.

Diagnostic tests are usually summarized in terms of sensitivity, specificity, AUC and LR. The advantages and limitations of these measures have been described earlier. ${ }^{26-28}$ LR appears to be higher than the previous measures in hierarchy; it expresses the magnitude by

Table 2 RNFL thickness parameters in glaucoma and healthy eyes with AUC and sensitivities at fixed specificities

\begin{tabular}{lccccccc}
\hline $\begin{array}{l}\text { RNFL thickness } \\
\text { (in microns) }\end{array}$ & $\begin{array}{c}\text { Normal subjects } \\
(\text { Mean } \pm \text { SD) }\end{array}$ & $\begin{array}{c}\text { Early glaucoma } \\
(\text { Mean } \pm \text { SD) }\end{array}$ & P-value & AUC & $95 \%$ CI & $\begin{array}{c}\text { Sensitivity at } \\
95 \% \text { specificity }\end{array}$ & $\begin{array}{c}\text { Sensitivity at } \\
80 \% \text { specificity }\end{array}$ \\
\hline Temporal quadrant & $75.46 \pm 8.47$ & $74.45 \pm 11.67$ & 0.53 & 0.537 & $0.439-0.630$ & $15.3 \%$ & $31.9 \%$ \\
Superior quadrant & $134.73 \pm 16.17$ & $117.97 \pm 20.39$ & $<0.001$ & 0.743 & $0.649-0.817$ & $33.0 \%$ & $60.4 \%$ \\
Nasal quadrant & $83.74 \pm 13.64$ & $70.02 \pm 11.87$ & $<0.001$ & 0.785 & $0.701-0.850$ & $31.9 \%$ & $65.9 \%$ \\
Inferior quadrant & $139.47 \pm 16.17$ & $113.18 \pm 22.48$ & $<0.001$ & 0.821 & $0.744-0.886$ & $58.2 \%$ & $73.6 \%$ \\
Average thickness & $108.42 \pm 9.87$ & $93.90 \pm 13.69$ & $<0.001$ & 0.799 & $0.718-0.870$ & $46.2 \%$ & $62.6 \%$ \\
\hline
\end{tabular}

Abbreviations: SD, standard deviation. 
Table 3 MIR parameters in glaucoma and healthy eyes with AUC and sensitivities at fixed specificities

\begin{tabular}{lccccccc}
\hline MIR parameters & $\begin{array}{c}\text { Normal } \\
(\text { Mean } \pm \text { SD })\end{array}$ & $\begin{array}{c}\text { Glaucoma } \\
(\text { Mean } \pm \text { SD) }\end{array}$ & P-value & AUC & 95\% CI & $\begin{array}{c}\text { Sensitivity at } \\
\text { 95\% specificity }\end{array}$ & $\begin{array}{c}\text { Sensitivity at } \\
80 \% \text { specificity }\end{array}$ \\
\hline Average $(\mu \mathrm{m})$ & $96.56 \pm 6.71$ & $88.04 \pm 10.52$ & $<0.001$ & 0.735 & $0.640-0.809$ & $37.4 \%$ & $60.4 \%$ \\
Superior $(\mu \mathrm{m})$ & $95.76 \pm 6.73$ & $88.62 \pm 10.87$ & $<0.001$ & 0.686 & $0.600-0.766$ & $31.9 \%$ & $54.9 \%$ \\
Inferior $(\mu \mathrm{m})$ & $97.36 \pm 7.20$ & $87.46 \pm 11.79$ & $<0.001$ & 0.745 & $0.658-0.818$ & $44.0 \%$ & $61.5 \%$ \\
SI average $(\mu \mathrm{m})$ & $-1.61 \pm 3.73$ & $1.16 \pm 8.44$ & $<0.001$ & 0.603 & $0.519-0.676$ & $35.2 \%$ & $47.3 \%$ \\
SI thickness SD & $10.45 \pm 4.74$ & $14.55 \pm 6.91$ & $<0.001$ & 0.728 & $0.644-0.808$ & $11.0 \%$ & $58.2 \%$ \\
FLV $(\%)$ & $0.44 \pm 1.19$ & $3.87 \pm 3.57$ & $<0.001$ & 0.908 & $0.855-0.945$ & $52.7 \%$ & $78.0 \%$ \\
GLV $(\%)$ & $2.61 \pm 4.54$ & $11.79 \pm 8.60$ & $<0.001$ & 0.874 & $0.802-0.923$ & $45.1 \%$ & $67.0 \%$ \\
RMS & $0.09 \pm 0.03$ & $0.13 \pm 0.06$ & $<0.001$ & 0.737 & $0.661-0.809$ & $28.6 \%$ & $57.1 \%$ \\
\hline
\end{tabular}

Abbreviations: SD, standard deviation; SI, superior minus inferior.

Table 4 Likelihood ratios (with $95 \%$ confidence interval) of the normative database classification of RNFL and MIR measurements with RTVue

\begin{tabular}{lccc}
\hline Parameter & $\begin{array}{c}\text { Within } \\
\text { normal limits }\end{array}$ & Borderline & $\begin{array}{c}\text { Outside } \\
\text { normal limits }\end{array}$ \\
\hline Average RNFL & 0.70 & 3.21 & 13.1 \\
thickness & $(0.60-0.81)$ & $(0.85-12.08)$ & $(4.10-41.7)$ \\
Superior RNFL & 0.83 & 4.58 & 21.1 \\
thickness & $(0.75-0.92)$ & $(1.30-16.17)$ & $(2.83-157)$ \\
Inferior RNFL & 0.63 & 1.45 & 22.3 \\
thickness & $(0.53-0.75)$ & $(0.70-3.01)$ & $(5.49-90.6)$ \\
Average MIR & 0.79 & 3.57 & 50.5 \\
thickness & $(0.71-0.89)$ & $(1.32-9.66)$ & $(3.08-830)$ \\
Superior MIR & 0.88 & 6.87 & 8.35 \\
thickness & $(0.80-0.96)$ & $(1.54-30.60)$ & $(1.90-36.7)$ \\
Inferior MIR & 0.71 & 4.12 & 72.6 \\
thickness & $(0.62-0.81)$ & $(1.15-14.80)$ & $(4.48-1178)$ \\
FLV & 0.53 & 1.65 & 4.89 \\
& $(0.42-0.67)$ & $(0.52-5.24)$ & $(2.82-8.48)$ \\
GLV & 0.64 & 1.92 & 6.07 \\
& $(0.53-0.77)$ & $(0.63-5.86)$ & $(2.96-12.4)$ \\
\hline
\end{tabular}

Abbreviations: RNFL, retinal nerve fiber layer; FLV, focal loss; GLV, global loss volume.

${ }^{\mathrm{a} A n a l y s i s ~ b a s e d ~ o n ~ n u m b e r ~ o f ~ e y e s . ~}$

which the probability of a diagnosis in a given patient is modified by the results of the test. In other words, the LR indicates how much a given diagnostic test result will raise or lower the pretest probability of the disease in question. We found that the outside normal limits results of most of the RNFL and MIR parameters had a large effect on the post-test probability of glaucoma. Borderline results were associated with small effects that would mean that borderline results were of little use in either ruling in or ruling out the diagnosis. Within normal limits, results were associated with no effect on the posttest probability of glaucoma, which would mean that, within normal limits, results were of little use in ruling out the diagnosis. However, it should be noted that even small effects on post-test probability may be relevant and useful, depending on other clinical information and the pretest probability of disease.

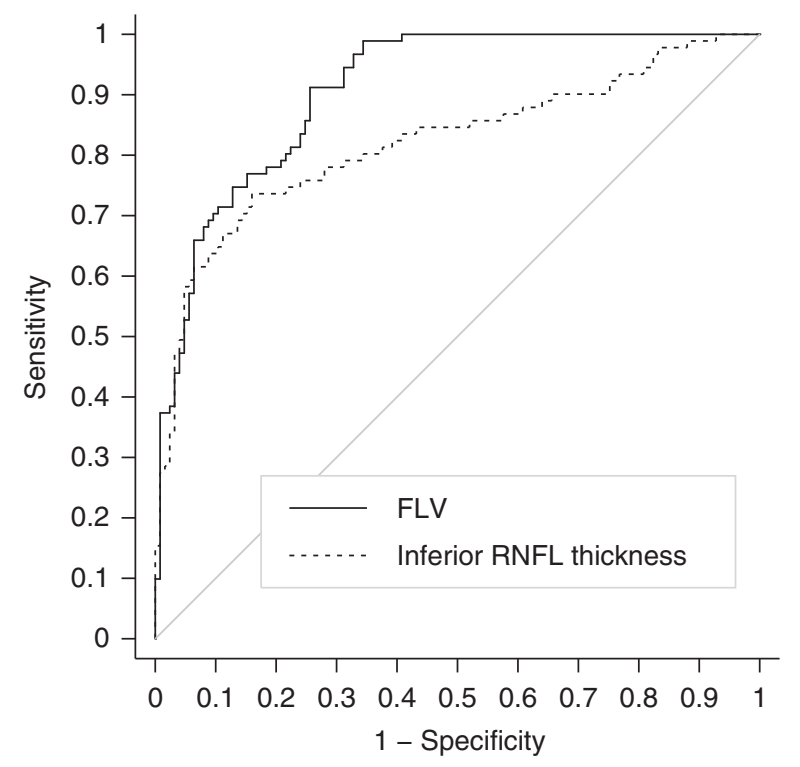

Figure 1 Receiver operating characteristic curves of the best retinal nerve fiber layer (inferior quadrant RNFL thickness) and macular inner retinal (focal loss volume, FLV) parameters.

Our results are in agreement with earlier studies comparing the diagnostic accuracies of RNFL and macular parameters with SD-OCT in early glaucoma. ${ }^{17,18}$ Seong et al ${ }^{18}$ in normal tension glaucoma eyes with early visual field damage, found that the diagnostic ability of MIR parameters were comparable to that of RNFL parameters; however, their analysis was limited to average, superior and inferior quadrant thicknesses of both RNFL and MIR. Using a regression model, Rao et $a l^{17}$ also found comparable diagnostic abilities between average RNFL and GCC RMS parameters at different severities of visual field damage. Mori et $a l^{12}$ also found that the MIR volume had diagnostic abilities comparable to that of RNFL measurements. In contrast, Huang et $a l^{29}$ in a similar study found that the MIR parameters were inferior compared with RNFL parameters to detect glaucoma. However, Huang et al used the earlier software version of RTVue and evaluated only the 


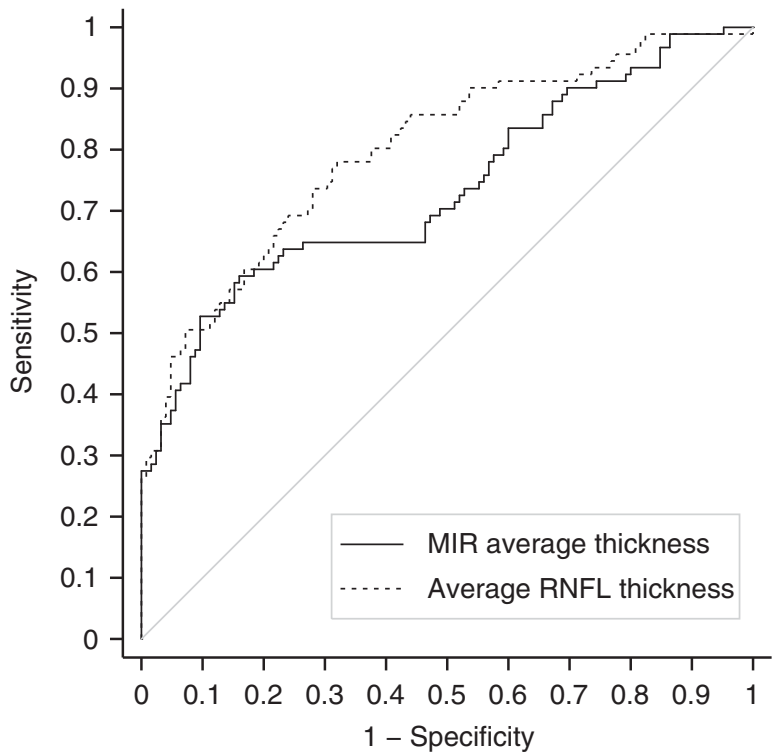

Figure 2 Receiver operating characteristic curves of the macular inner retinal average thickness and average retinal nerve fiber layer thickness parameters.

superior, inferior, and average MIR parameters and not FLV, GLV, or RMS that were in fact, the MIR parameters associated with higher AUCs both in our study and in previous studies by Seong et al and Rao et al.

These results of improved diagnostic abilities of macular evaluation algorithms with SD-OCT compared with Stratus OCT may be attributable to the better resolution and greater speed of image acquisition of SD-OCT. The limited axial resolution of the Stratus OCT allowed for only full macula thickness measurements, whereas the higher resolution of SD-OCT allows for more specific segmentation of the retinal layers with which layers associated with glaucomatous damage, namely nerve fiber layer, ganglion cell layer and inner plexiform layer can be studied and analyzed. The greater scanning speed of SD-OCT enables acquisition of a large number of scans in a single imaging session, reducing the need for data interpolation.

One of the limitations of the study is the case-control design with a clear distinction between glaucoma patients (cases) defined, based on the presence of glaucomatous visual field defects, and normal subjects (controls) recruited from the general population and having no suspicious findings of glaucoma. Such a design has been shown to inflate the actual diagnostic ability of a test..$^{30,31}$ Another limitation, in general to all studies in glaucoma is the lack of reference standard for diagnosing the disease. Our glaucoma cohort included patients with evidence of both structural changes in optic disc and correlating visual field changes. Although the strict inclusion criteria would have reduced the false positives to a great extent, this would have excluded a few cases of glaucoma with damage detectable only on structural or functional tests. However, as the aim of our study was to compare RNFL and MIR parameters of SD-OCT in the same population, this is unlikely to have influenced the comparison.

In conclusion, MIR parameters with SD-OCT were as good as the RNFL parameters to detect early glaucoma in Indian eyes. Further studies should evaluate if our study results are reproducible in other ethnic groups.

\section{Summary}

What was known before

- SD-OCT parameters have good diagnostic abilities to discriminate glaucomatous eyes from normal eyes.

- Macular parameters have similar diagnostic abilities compared with RNFL parameters in glaucoma. But these studies included the entire range of disease severity and were largely restricted to Caucasian population.

What this study adds

- Diagnostic abilities of macular parameters are as good as RNFL parameters in Indian eyes with early glaucoma.

\section{Conflict of interest}

Drs Rao and Garudadri are consultants to Allergan. Dr Garudadri is also a consultant to Merck and Alcon. Dr Garudadri received RTVue as a research grant from Optovue for Indian normative database collection. The other manuscript authors have no financial interest in any of the products mentioned in the study.

\section{Acknowledgements}

Dr Garudadri received RTVue as a research grant from Optovue for Indian normative database collection.

\section{References}

1 Medeiros FA, Zangwill LM, Bowd C, Vessani RM, Susanna Jr R, Weinreb RN. Evaluation of retinal nerve fiber layer, optic nerve head, and macular thickness measurements for glaucoma detection using optical coherence tomography. Am J Ophthalmol 2005; 139: 44-55.

2 Wollstein G, Ishikawa H, Wang J, Beaton SA, Schuman JS Comparison of three optical coherence tomography scanning areas for detection of glaucomatous damage. Am J Ophthalmol 2005; 139: 39-43.

3 Hougaard JL, Heijl A, Bengtsson B. Glaucoma detection by Stratus OCT. J Glaucoma 2007; 16: 302-306.

4 Parikh RS, Parikh S, Sekhar GC, Kumar RS, Prabakaran S, Babu JG et al. Diagnostic capability of optical coherence 
tomography (Stratus OCT 3) in early glaucoma. Ophthalmology 2007; 114: 2238-2243.

5 Nouri-Mahdavi K, Nikkhou K, Hoffman DC, Law SK, Caprioli J. Detection of early glaucoma with optical coherence tomography (StratusOCT). J Glaucoma 2008; 17: $183-188$

6 Nassif N, Cense B, Park B, Pierce M, Yun S, Bouma B et al. In vivo high-resolution video-rate spectral-domain optical coherence tomography of the human retina and optic nerve. Opt Express 2004; 12: 367-376.

7 Wojtkowski M, Srinivasan V, Ko T, Fujimoto J, Kowalczyk A Duker J. Ultrahigh-resolution, high-speed, Fourier domain optical coherence tomography and methods for dispersion compensation. Opt Express 2004; 12: 2404-2422.

8 Zeimer R, Asrani S, Zou S, Quigley H, Jampel H. Quantitative detection of glaucomatous damage at the posterior pole by retinal thickness mapping. A pilot study. Ophthalmology 1998; 105: 224-231.

9 Curcio CA, Allen KA. Topography of ganglion cells in human retina. J Comp Neurol 1990; 300: 5-25.

10 Tan O, Chopra V, Lu AT, Schuman JS, Ishikawa H, Wollstein G et al. Detection of macular ganglion cell loss in glaucoma by Fourier-domain optical coherence tomography. Ophthalmology 2009; 116: 2305-2314, e2301-2302.

11 Rao HL, Zangwill LM, Weinreb RN, Sample PA, Alencar LM, Medeiros FA. Comparison of different spectral domain optical coherence tomography scanning areas for glaucoma diagnosis. Ophthalmology 2010; 117: 1692-1699, 1699, e1691.

12 Mori S, Hangai M, Sakamoto A, Yoshimura N. Spectraldomain optical coherence tomography measurement of macular volume for diagnosing glaucoma. J Glaucoma 2010; 19: $528-534$.

13 Garas A, Vargha P, Hollo G. Diagnostic accuracy of nerve fibre layer, macular thickness and optic disc measurements made with the RTVue-100 optical coherence tomograph to detect glaucoma. Eye (Lond) 2011; 25: 57-65.

14 Schulze A, Lamparter J, Pfeiffer N, Berisha F, Schmidtmann I, Hoffmann EM. Diagnostic ability of retinal ganglion cell complex, retinal nerve fiber layer, and optic nerve head measurements by Fourier-domain optical coherence tomography. Graefes Arch Clin Exp Ophthalmol 2011; 249: 1039-1045.

15 Medeiros FA, Zangwill LM, Bowd C, Sample PA, Weinreb RN. Influence of disease severity and optic disc size on the diagnostic performance of imaging instruments in glaucoma. Invest Ophthalmol Vis Sci 2006; 47: 1008-1015.

16 Garudadri CS, Rao HL, Parikh RS, Jonnadula GB, Selvaraj P, Nutheti $R$ et al. Effect of Optic Disc Size and Disease Severity on the Diagnostic Capability of Glaucoma Imaging Technologies in an Indian Population. J Glaucoma 2011; e-pub ahead of print 25 April 2011; doi:10.1097/ IJG.0b013e31821829f1.

17 Rao HL, Leite MT, Weinreb RN, Zangwill LM, Alencar LM, Sample PA et al. Effect of disease severity and optic disc size on diagnostic accuracy of RTVue spectral domain optical coherence tomograph in glaucoma. Invest Ophthalmol Vis Sci 2011; 52: 1290-1296.

18 Seong M, Sung KR, Choi EH, Kang SY, Cho JW, Um TW et al. Macular and peripapillary retinal nerve fiber layer measurements by spectral domain optical coherence tomography in normal-tension glaucoma. Invest Ophthalmol Vis Sci 2010; 51: 1446-1452.

19 Hodapp E PR, Anderson DR (eds). Clinical Decisions in Glaucoma. St. Louis, 1993; pp 53.

20 Zhou X-H ON, McClish DK. Analysis of correlated ROC data. In: Zhou X-H, Obuchowski NA, McClish DK (eds). Statistical Methods in Diagnostic Medicine. John Wiley \& Sons, Inc: New York, 2002; pp 274-306.

21 Alonzo TA, Pepe MS. Distribution-free ROC analysis using binary regression techniques. Biostatistics 2002; 3: 421-432.

22 Jaeschke R, Guyatt GH, Sackett DL. Users' guides to the medical literature. III. How to use an article about a diagnostic test. B. What are the results and will they help me in caring for my patients? The Evidence-Based Medicine Working Group. JAMA 1994; 271: 703-707.

23 Radack KL, Rouan G, Hedges J. The likelihood ratio. An improved measure for reporting and evaluating diagnostic test results. Arch Pathol Lab Med 1986; 110: 689-693.

24 Fagan TJ. Letter: Nomogram for Bayes theorem. $N$ Engl J Med 1975; 293: 257.

25 Simel DL, Samsa GP, Matchar DB. Likelihood ratios with confidence: sample size estimation for diagnostic test studies. J Clin Epidemiol 1991; 44: 763-770.

26 Langlotz CP. Fundamental measures of diagnostic examination performance: usefulness for clinical decision making and research. Radiology 2003; 228: 3-9.

27 Medeiros FA, Zangwill LM, Bowd C, Weinreb RN. Comparison of the GDx VCC scanning laser polarimeter, HRT II confocal scanning laser ophthalmoscope, and stratus OCT optical coherence tomograph for the detection of glaucoma. Arch Ophthalmol 2004; 122: 827-837.

28 Zangwill LM, Jain S, Racette L, Ernstrom KB, Bowd C, Medeiros FA et al. The effect of disc size and severity of disease on the diagnostic accuracy of the Heidelberg Retina Tomograph Glaucoma Probability Score. Invest Ophthalmol Vis Sci 2007; 48: 2653-2660.

29 Huang JY, Pekmezci M, Mesiwala N, Kao A, Lin S. Diagnostic Power of Optic Disc Morphology, Peripapillary Retinal Nerve Fiber Layer Thickness, and Macular Inner Retinal Layer Thickness in Glaucoma Diagnosis With Fourier-domain Optical Coherence Tomography. J Glaucoma 2011; 20: 87-95.

30 Lijmer JG, Mol BW, Heisterkamp S, Bonsel GJ, Prins MH, van der Meulen $\mathrm{JH}$ et al. Empirical evidence of designrelated bias in studies of diagnostic tests. JAMA 1999; 282: 1061-1066.

31 Medeiros FA, Ng D, Zangwill LM, Sample PA, Bowd C, Weinreb RN. The effects of study design and spectrum bias on the evaluation of diagnostic accuracy of confocal scanning laser ophthalmoscopy in glaucoma. Invest Ophthalmol Vis Sci 2007; 48: 214-222. 